\title{
Effects of pacemaker and implantable cardioverter defibrillator electrodes on tricuspid regurgitation and right sided heart functions
}

\author{
Peyman Arabi ${ }^{1}$, Necla Özer ${ }^{1}$, Ahmet Hakan Ateş ${ }^{2}$, \\ Hikmet Yorgun $^{1}$, Ali Oto ${ }^{1}$, Kudret Aytemir ${ }^{1}$ \\ ${ }^{1}$ Department of Cardiology, Faculty of Medicine, Hacettepe University, Ankara, Turkey \\ ${ }^{2}$ Samsun Mehmet Aydın Training and Research Hospital, Samsun, Turkey
}

\begin{abstract}
Background: The aim of this study was to assess the effect of trans-tricuspid placement of permanent pacemaker (PPM), implantable cardioverter defibrillator (ICD) and cardiac resynchronization therapy (CRT) leads prospectively on tricuspid valve and right-sided heart functions using two-dimensional echocardiography.

Methods: A total of 41 patients (31 male, mean age: $63.6 \pm 12.2$ years) were included in this prospective study. Initial echocardiographic evaluation was performed before cardiac device implantation and re-evaluation by echocardiography was performed immediately after the procedure and at $1^{\text {st }}, 6^{\text {th }}$ and $12^{\text {th }}$ months. In addition to standard echocardiographic examinations, vena contracta (VC), proximal isovelocity surface area (PISA), and tissue Doppler evaluations were also performed in the study population.
\end{abstract}

Results: Tricuspid regurgitation (TR) is worsened by 1 grade in $70.8 \%$ of the patients and 2 grades in $17.1 \%$ of the patients in the follow-up. Eight patients without baseline TR developed new-onset TR (9.8\% mild, 9.8\% moderate) after lead implantation. In the follow-up period, $41.5 \%$ of the patients who had mild TR before lead implantation developed moderate TR and 7.3\% developed severe TR, whereas $19.5 \%$ of the patients with moderate TR developed severe $T R$ during the follow-up. In the follow-up period, VC of TR was increased [median: 0.32 $(0.16-0.60) \mathrm{cm}$ in pre-implantation period, and $0.41(0.18-0.80) \mathrm{cm}$ at $12^{\text {th }}$ month, $\left.p=0.001\right]$. Similarly PISA value of TR was also increased [median: $0.46(0.15-1.10) \mathrm{cm}$ in pre-implantation period and $0.52(0.28-1.20) \mathrm{cm}$ at $12^{\text {th }}$ month, $\left.p=0.001\right]$. However, there is not a significant difference between PPMs/ICDs and CRTs regarding the effects on $T R(p<0.05)$. In addition, right ventricular dimensions and right atrial volumes were increased during the follow-up.

Conclusions: Implantation of permanent transvenous right ventricular electrode is associated with worsening of TR, right atrial and right ventricular dimensions. Further studies are needed in order to both outline the effect of those findings on outcomes and clarify the time dependent changes in those functions. (Cardiol J 2015; 22, 6: 637-644)

Key words: tricuspid regurgitation, implantable cardiac devices, right heart functions, permanent pacemaker, implantable cardioverter defibrillator

Address for correspondence: Prof. Necla Özer, Hacettepe University, Faculty of Medicine, Department of Cardiology, Ankara, Turkey, tel: 090312 3051780, fax: 090312 3055000, e-mail: neclaozer@gmail.com

Received: 17.05.2015 Accepted: 26.08.2015 


\section{Introduction}

Implantable cardioverter defibrillators (ICDs) and permanent pacemakers (PPMs) are commonly used devices for the treatment of cardiac conduction disorders and life threatening arrhythmias in the current practice. With the advent of newer technologies and implantation techniques, the number of implanted devices has been increased all over the world [1]. Nevertheless, implantation of these devices is an invasive procedure and which is associated with several early and late complications. Despite the most stressed periprocedural complications relevant mainly to cardiac device, tricuspid regurgitation (TR) due to right ventricle (RV) endocardial lead implantation is a commonly observed but mostly an overlooked condition.

Tricuspid regurgitation is a frequently observed valvular disease and the mechanism of TR is mostly due to the secondary causes resulting in $\mathrm{RV}$ and tricuspid annular dilatation [2]. However, there are several proposed mechanisms causing TR and RV dysfunction after lead implantation. Endocardial lead implantation can cause structural TR due to deformity of valve including adherence and impingement of electrodes to valvular structures or perforation of valve and its components $[3,4]$. In addition, high percentage of RV pacing might cause TR due to atrioventricular discordance with unicameral ventricular pacing mode. Moreover, recurrent thrombus embolization from cardiac leads might also cause pulmonary hypertension and TR due to RV dilatation [5].

Current knowledge regarding the effects of cardiac leads on tricuspid valve functions largely based on retrospective or observational studies. Moreover, there is paucity of data about the effects of lead implantation on right-sided heart functions especially evaluated by more quantitative methods. In the light of those data, in this prospective study, we aimed to evaluate the prevalence and severity of TR and effect of trans-tricuspid lead implantation on both tricuspid valve and right sided heart chambers' functions, using 2-dimensional and Doppler echocardiography in a group of patients referred for PPM, ICD or cardiac resynchronization therapy (CRT) implantation.

\section{Methods}

\section{Study population}

In this prospective study, we enrolled a consecutive of subset patients who had transtricuspid implanted PPM/ICD or CRT leads according to the indications as assessed by guidelines [6]. Patients with severe stenosis or regurgitation in atrioventricular or semilunar valves and a history of previous tricuspid valve repair were excluded from the analysis. All of the patients were evaluated with 2 dimensional (2D)-echocardiography before and 3 times after the cardiac device implantation $\left(1^{\text {st }}, 6^{\text {th }}\right.$ and $12^{\text {th }}$ months after the procedure).

Medical history of hypertension, hypercholesterolemia, coronary artery disease (CAD), diabetes mellitus (DM), chronic obstructive pulmonary disease (COPD), family history of CAD and current medications were systematically evaluated. Pre- and post-implantation data of the leads were all collected from the study population. All RV leads of PM and ICD's were implanted in the RV apex and left ventricular (LV) electrodes of CRT in the posterolateral branch of coronary sinus. In our study group, we used pacemaker, ICD and CRT of St. Jude family; DDD Zephyr (St. Jude Medical, MN, USA) for pacemaker, Fortify Assura ICD (St. Jude Medical, USA) for DDD-ICD and Unify Quadra or Quadra Assura models (St. Jude Medical, USA) for CRT-D. The PM/ICD settings were individually programmed based on the indication for cardiac devices. In order to decrease the detrimental effects of RV pacing, atrioventricular interval was lengthened in appropriate patients based on the indication of implantation. The target percentage of CRT stimulation was $>95 \%$ in the study population which was achieved in all patients. All devices were interrogated in our center at the time of routine control visits $\left(1^{\text {st }}, 6^{\text {th }}\right.$ and $12^{\text {th }}$ months) after the procedure. Informed consent was obtained from each patient and this study was approved by the local Ethics Committee.

\section{Echocardiographic evaluation}

Standard echocardiographic imaging was performed in the left lateral decubitus position in the parasternal and apical views. Two-dimensional, M-mode, pulsed and color flow Doppler echocardiographic examinations of all subjects were performed by the same examiner with a commercially available machine (Vingmed System Five GE ultrasound, Horten, Norway, $2.5-3.5 \mathrm{mHz}$ phased array transducer) who was blinded to the clinical details of the subjects in the study group. During echocardiography, a 1-lead electrocardiogram was recorded continuously. Left ventricular enddiastolic (LVEDD), left ventricular end-systolic (LVESD), RV size and right atrial (RA) major and minor dimensions were measured from parasternal long-axis views and apical views according to the 
standards of the American Society of Echocardiography [7]. The ejection fraction of RV and LV was measured according to the guidelines by using modified Simpson method [8]. Right atrial maximum and minimum diameters and three different $\mathrm{RV}$ size $\left(\mathrm{RV}_{1}\right.$, on the level of tricuspid annulus; $\mathrm{RV}_{2}$, on level of mid-ventricular part and $\mathrm{RV}_{3}$, longitudinal dimension from apex to annulus) were also measured [8]. Morphological and functional features of aortic, mitral, tricuspid valve and pulmonary valves were analyzed according to generally accepted guidelines and TR was graded as absent, trace, mild, moderate and severe based on the jet area by color Doppler.

Tricuspid inflow indices were obtained by pulsed-wave Doppler from the apical 4-chamber view to assess RV filling. The tricuspid annular plane systolic excursion (TAPSE) examination for RV function and vena contracta (VC) width, and proximal isovelocity surface area (PISA) measurements for TR were performed in all participants according to the recommended guidelines [8]. The degree of TR was classified as mild, moderate and severe using $\mathrm{VC}$ width $(\mathrm{VC}<0.3 \mathrm{~cm}$ for mild TR, VC $0.3-0.7$ for moderate TR and VC $>0.7$ for severe TR) and PISA (PISA $<0.5 \mathrm{~cm}$ for mild TR; PISA $0.5-0.9 \mathrm{~cm}$ for moderate TR and PISA $>0.9 \mathrm{~cm}$ for severe TR) [9].

Tissue Doppler imaging echocardiography was performed by transducer frequencies of $3.5-4.0 \mathrm{MHz}$, adjusting the spectral pulsed Doppler signal filters until a Nyquist limit of $15-20 \mathrm{~cm} / \mathrm{s}$ was reached and using the minimal optimal gain. The monitor sweep speed was set at $50-100 \mathrm{~mm} / \mathrm{s}$ to optimize the spectral display of myocardial velocities. The pulsed-wave tissue Doppler imaging was performed in the apical views by placing a $3 \mathrm{~mm}$ sample volume at the level of left ventricular lateral mitral annulus, septal mitral annulus, and RV tricuspid annulus. The sampling window was positioned as parallel as possible with the myocardial segment of interest to ensure the optimal angle of imaging. Peak systolic (S'), early (E') and late diastolic myocardial velocities (A') were recorded. Several cardiac cycles were evaluated and the best three consecutive ones were analyzed and averaged.

\section{Follow-up}

In the follow-up period, clinical assessment, cardiac device evaluation and echocardiographic examination were all performed. Echocardiographic examination was repeated after $1^{\text {st }}, 6^{\text {th }}$ and $12^{\text {th }}$ months in the follow-up period. All of the patients included in the analysis completed the follow-up controls.

\section{Statistical analysis}

Statistical analyses were made using Statistical Package for Social Sciences (SPSS) (IBM SPSS Inc. Chicago, IL) and Medcalc 11.4.2. (Medcalc Software, Mariakerke, Belgium). Normally distributed continuous parameters were presented as mean \pm \pm standard deviation and skewed continuous parameters were expressed as median (interquartile range defined as Q1-Q3). Categorical data were presented as frequencies and percentages and were compared using $\chi^{2}$ test. Comparisons between baseline characteristics were performed with independent student's t, Mann-Whitney rank-sum, Fisher exact or $\chi^{2}$ tests where appropriate. The change in the follow-up period was evaluated by using ANOVA in normally distributed data and Friedman test in abnormally distributed data. A 2 -tailed $\mathrm{p}<0.05$ was considered statistically significant.

\section{Results}

\section{Basal clinical and echocardiographic characteristics}

A total of 41 patients (31 male, $75 \%$ ) with a mean age of $63.6 \pm 12.2$ years were enrolled in the analysis. Among the study population, $61 \%$ had hypertension, $36.6 \%$ had DM and $7.3 \%$ had COPD. A total of 41 cardiac devices were implanted and $25(61 \%)$ were DDD ICD, $9(22 \%)$ of them were CRT-D and 7 (17\%) were DDD PM. Among those patients, $1(2.5 \%)$ patient had aortic valve replacement (AVR), 1 (2.5\%) patient had mitral valve replacement (MVR) and 1 (2.5\%) patient had both AVR and MVR in the past medical history. The baseline characteristics of the patients are described in Table 1.

Before PM implantation all patients had complete echocardiographic examinations including both LV and RV functions. The mean ejection fraction, LVEDD and LVESD of the study group were $34.2 \pm 13.1 \%, 60.2 \pm 9.6 \mathrm{~mm}$ and $47.8 \pm$ $\pm 11.2 \mathrm{~mm}$, respectively. In the study group, trace-mild TR was found in $31(75 \%)$ patients and moderate TR was found in only $10(25 \%)$ patients. The baseline TAPSE value of the study group was $18.9 \pm 3.4 \mathrm{~mm}$ and the mean pulmonary artery pressure (PAP) was $35.5 \pm 3.7 \mathrm{~mm} \mathrm{Hg}$. The tissue Doppler parameters measured from tricuspid annulus were $7.5 \pm 3.1 \mathrm{~cm} / \mathrm{s}$ for $\mathrm{E} ; 11.7 \pm 4.1 \mathrm{~cm} / \mathrm{s}$ for $\mathrm{A}$ and $9.5 \pm 2.2 \mathrm{~cm} / \mathrm{s}$ for S. During the follow-up period, statistically signifi- 
Table 1. Baseline characteristics of the study population.

\begin{tabular}{lc}
\hline Variable & $\begin{array}{c}\text { Study population } \\
\text { (n }=41)\end{array}$ \\
\hline Age [years] & $63.6 \pm 12.2$ \\
Male gender & $31(75.6 \%)$ \\
Body mass index $\left[\mathrm{kg} / \mathrm{m}^{2}\right]$ & $28.5 \pm 3.3$ \\
Hypertension & $25(61 \%)$ \\
Diabetes mellitus & $15(36.6 \%)$ \\
COPD & $3(7.3 \%)$ \\
CAD & $32(78 \%)$ \\
Hyperlipidemia & $34(82.9 \%)$ \\
Medications & \\
Beta-blockers & $38(92.7 \%)$ \\
ACE/ARB & $31(75.6 \%)$ \\
Diuretics & $20(48.8 \%)$ \\
Acetylsalycyclic acid & $31(75.6 \%)$ \\
Statin & $26(63.4 \%)$ \\
Previous cardiac operation & \\
AVR & $1(2.4 \%)$ \\
AVR + MVR & $1(2.4 \%)$ \\
MVR & $1(2.4 \%)$ \\
Cardiac device types & $35.5 \pm 3.7$ \\
CRT-D & $9(21.9 \%)$ \\
DDD & $7(17 \%)$ \\
DDD-ICD & $25(61 \%)$ \\
Tricuspid regurgitation severity & \\
Absent-minimal & $8(19.5 \%)$ \\
Mild & $23(56.1 \%)$ \\
Moderate & $10.4 \%)$ \\
Pevere & \\
\hline ACE (mm Hg] & \\
\hline
\end{tabular}

ACE - angiotensin converting enzyme; ARB - angiotensin receptor blocker; AVR - aortic valve replacement; CAD - coronary artery disease; COPD - chronic obstructive pulmonary disease CRTD - cardiac resynchronization therapy with defibrillator; ICD - implantable cardioverter defibrillator; MVR — mitral valve replacement; PAP — pulmonary artery pressure cant difference was not observed regarding TAPSE, PAP or tissue Doppler parameters ( $>>0.05$ ) (Table 2). Moreover, those parameters did not show statistical difference when the device type considered.

\section{Effect of lead implantation on TR measured by color flow jet}

During the 1-year follow-up, a statistically significant increase in TR was observed in the study population which is independent from the lead type implanted ( $\mathrm{p}=0.001)$. Before device implantation $8(19.5 \%)$ patients were free of TR, $23(56.1 \%)$ patients had trace-mild TR and $10(24.4 \%)$ patients had moderate TR. After device implantation mild TR was developed in $4(9.8 \%)$ patients and moderate TR was developed in $4(9.8 \%)$ patients who did not have any TR before device implantation $(\mathrm{p}<0.05)$. Among the 23 patients having trace-mild TR at the beginning of the study, 17 (41.5\%) developed moderate TR and $3(7.3 \%)$ patients developed severe TR $(p<0.05)$ during the follow-up period. Similarly, there was an increase in the grade of TR also among 10 patients having moderate TR before lead implantation. While $8(19.5 \%)$ of those patients developed severe TR in the follow-up period, 2 patients remained in the moderate TR grade after lead implantation during the follow-up $(\mathrm{p}<0.05)$.

As a result, after lead implantation, compared to basal levels, TR was worsened by at least 1 grade in $70.8 \%$ of the study population and 2 grades in $17.1 \%$ of the whole study population during the follow-up. The change in TR grade was observed at the $1^{\text {st }}$ month of the follow-up when compared to preimplantation period $(\mathrm{p}=0.001)$, however difference in TR grade was not significant between $1^{\text {st }}$ month and $6^{\text {th }}-12^{\text {th }}$ months values in all device types (Fig. 1).

Table 2. The change in the tissue Doppler parameters, tricuspid annular plane systolic excursion, pulmonary artery pressure, and inferior vena cava during the follow-up ( $p$ value indicating the significance within group).

\begin{tabular}{lccccc}
\hline Variable & Post-implantation & $\mathbf{1}^{\text {st }}$ month & $\mathbf{6}^{\text {th }}$ month & $\mathbf{1 2}^{\text {th }}$ month & P \\
\hline TAPSE $(\mathrm{mm}]$ & $18.93 \pm 3.43$ & $18.88 \pm 3.21$ & $18.46 \pm 3.45$ & $18.34 \pm 3.79$ & 0.213 \\
E$^{\prime}[\mathrm{cm} / \mathrm{s}]$ & $7(3-16)$ & $8(3-17)$ & $8.1(4-14)$ & $7.7(3-16)$ & 0.353 \\
$\mathrm{~A}^{\prime}[\mathrm{cm} / \mathrm{s}]$ & $11(4.5-23)$ & $12(4-26)$ & $10(3-24)$ & $12(3-25)$ & 0.270 \\
$\mathrm{~S}^{\prime}[\mathrm{cm} / \mathrm{s}]$ & $9.9(4.6-20)$ & $10(5.7-18)$ & $9.786-17)$ & $9.5(4-19)$ & 0.691 \\
PAP $[\mathrm{mm} \mathrm{Hg}]$ & $35(20-75)$ & $35(25-70)$ & $35(25-85)$ & $35(20-75)$ & 0.420 \\
IVC size $[\mathrm{mm}]$ & $15.71 \pm 2.94$ & $16.17 \pm 2.77$ & $16.39 \pm 2.91$ & $16.61 \pm 2.64$ & 0.085 \\
\hline
\end{tabular}

IVC — inferior vena cava; PAP — pulmonary artery pressure; TAPSE — tricuspid annular plane systolic excursion 


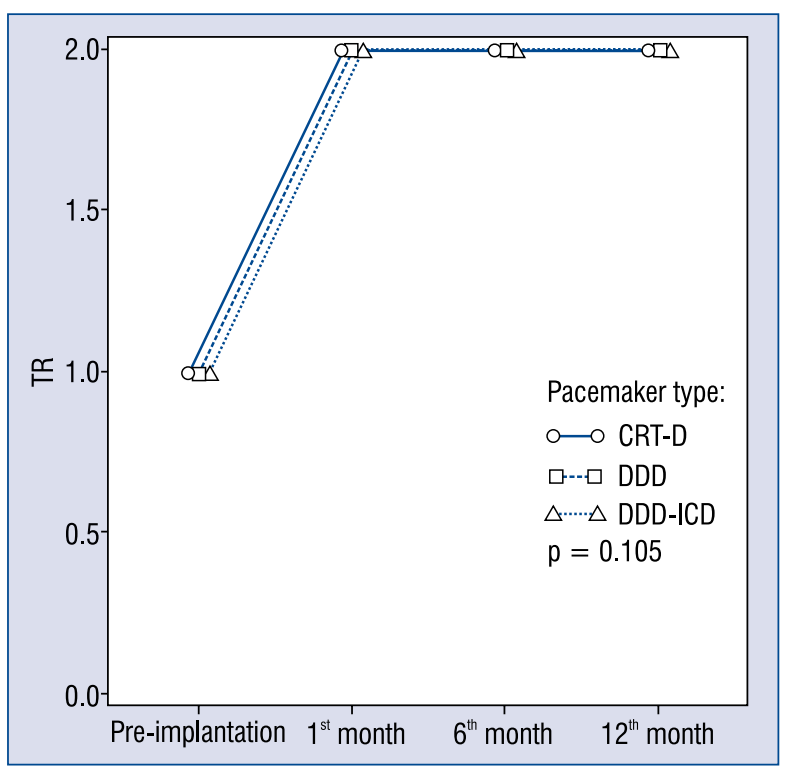

Figure 1. Change in tricuspid regurgitation (TR) between cardiac device types assessed by color flow jet.

\section{Effect of lead implantation on TR measured by semiquantitative and quantitative methods}

In the follow-up period, VC of TR was increased significantly and progressively in every follow-up period (median: 0.32 [0.16-0.60] $\mathrm{cm}$ in pre-implantation period, and $0.41[0.18-0.80] \mathrm{cm}$ at $12^{\text {th }}$ month, $\left.\mathrm{p}=0.001\right)$. Similarly, PISA value was also increased in the follow-up period for TR (median: $0.46[0.15-1.10] \mathrm{cm}$ in pre-implantation period and $0.52[0.28-1.20] \mathrm{cm}$ at $12^{\text {th }}$ month, $\mathrm{p}=0.001$ ) as shown in Table 3 . However, there was no statistically significant difference between implanted lead types and VC and PISA values of the TR between during the follow-up period $(\mathrm{p}=0.074$ and $\mathrm{p}=$ $=0.192$, respectively). The trend of change in PISA and VC values were shown in Figure 2.

\section{Effect of lead implantation on RA and RV dimensions and functions}

When compared to basal values, $\mathrm{RV}$ diameters showed progressive increase after cardiac device implantation at the $12^{\text {th }}$ month. In the study group, $\mathrm{RV}_{1}, \mathrm{RV}_{2}$ and $\mathrm{RV}_{3}$ dimensions were as follows: $3.5 \pm 0.3 \mathrm{~cm}$ vs. $3.7 \pm 0.3 \mathrm{~cm} ; 2.9 \pm 0.3 \mathrm{~cm}$ vs. $3.0 \pm 0.3 \mathrm{~cm} ; 7.5 \pm 0.7$ vs. $7.7 \pm 0.7 \mathrm{~cm}$, respectively ( $<<0.001)$. Similar to the TR, the change in $\mathrm{RV}$ diameters did not differ between cardiac device types. Among RA maximum and minimum diameters, RA maximum diameter did not change at $12^{\text {th }}$ month significantly when compared to basal value $(51.4 \pm 8.5 \mathrm{~cm}$ vs. $51.9 \pm 9.4 \mathrm{~cm}, \mathrm{p}=0.486)$

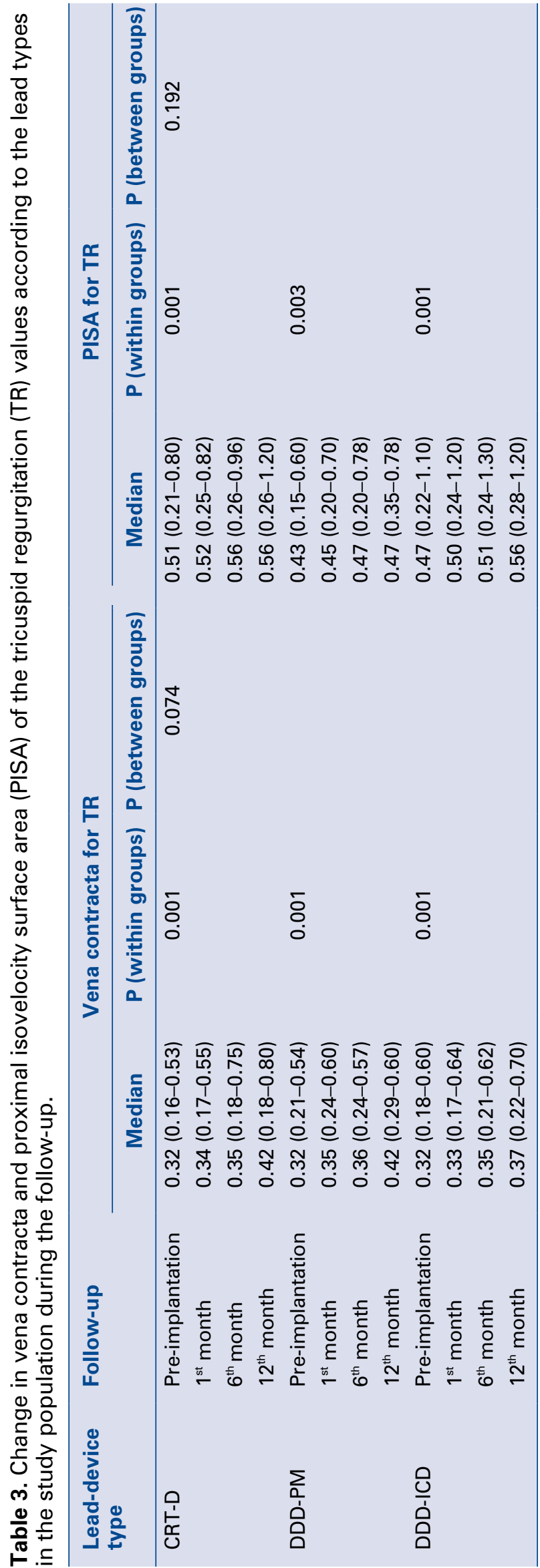




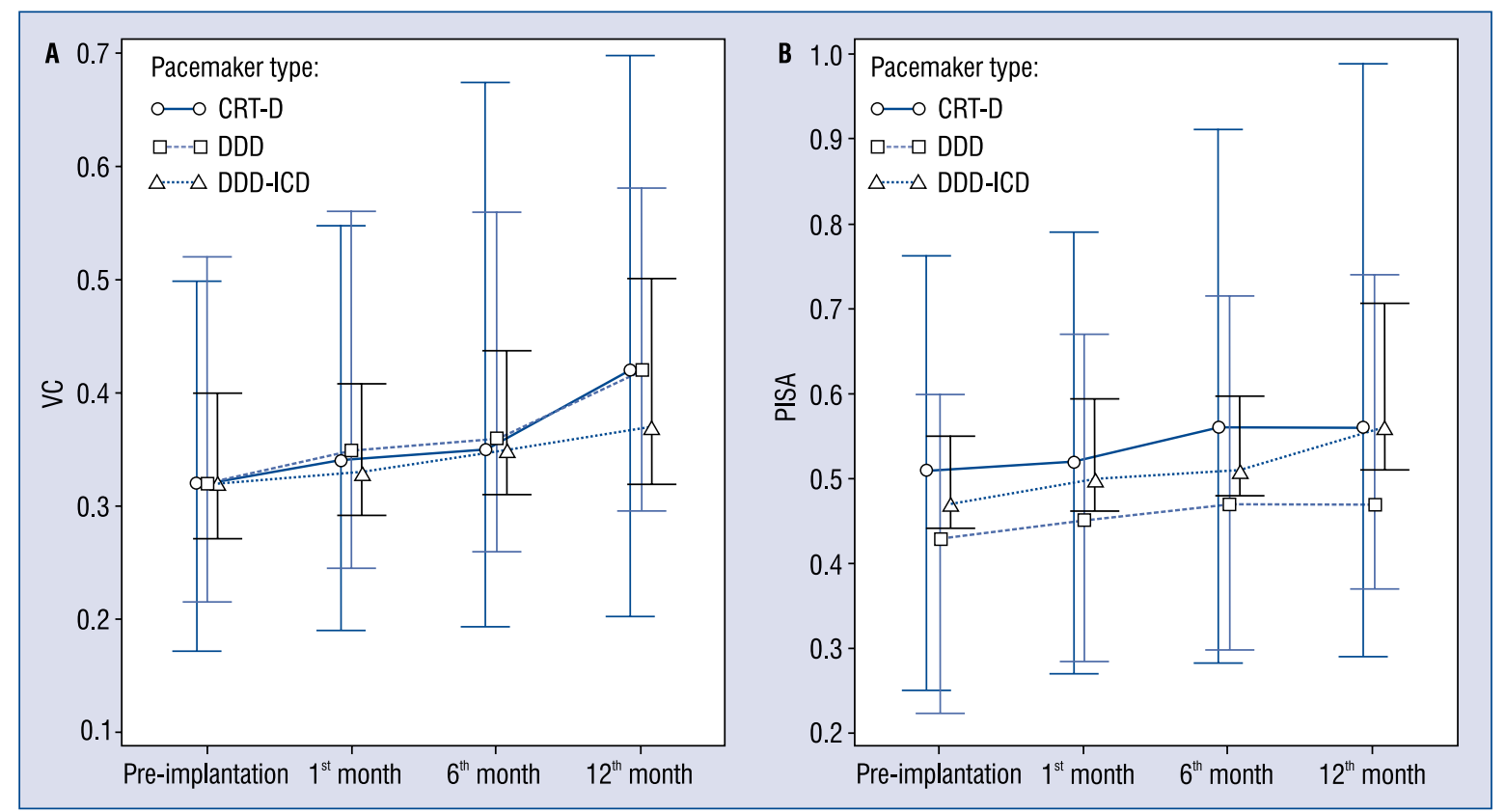

Figure 2. Change in vena contracta (VC) (A) and proximal isovelocity surface area (PISA) (B) during the follow-up compared to basal levels for all cardiac device types $\left(p<0.005\right.$ between preimplantation and $12^{\text {th }}$ month for both VC and PISA).

however RA minimum diameter showed significant increase compared to baseline value $(40.4 \pm 8.7 \mathrm{~cm}$ vs. $43.1 \pm 7.6 \mathrm{~cm}, \mathrm{p}<0.05)$.

In addition to the changes in RV dimensions, ICD/PPM implantation was also associated with worsening in RV ejection fraction. Compared to preimplantation values, $\mathrm{RV}$ ejection fraction decreased significantly throughout the follow-up period irrespective of the device type implanted $(39.9 \pm 10.1 \%$ at pre-implantation period, $36.4 \pm$ $\pm 9.6 \%$ at $1^{\text {st }}$ month, $32.7 \pm 9.5 \%$ at $6^{\text {th }}$ month and $29.7 \pm 9.9 \%$ at the $12^{\text {th }}$ month, $\left.\mathrm{p}<0.001\right)$.

\section{Discussion}

With the advent of cardiac device implantation via endocardial route, many researchers proposed that PM/ICD electrodes worsen tricuspid insufficiency as they pass through the tricuspid orifice $[3,10]$. However, to the best of our knowledge, there is not any prospective study in the literature which evaluated the effects of PM/ICD lead implantation via endocardial route on both tricuspid valve and right chamber functions. In this study, our findings indicated that PM/ICD/CRT-D implantation causes an increase in tricuspid insufficiency and deterioration in RA and RV dimensions and decrease in RV functions.

Evaluation of right heart chambers is different from the left side where most of the investiga- tors were more familiar compared to right side. The RV has a complex anatomy and a nonplanar annulus. The $2 \mathrm{D}$ echocardiography necessitates multiple views to assess the tricuspid valve and its functions. However, the exact mechanism of TR due to electrode damage was diagnosed with transesophageal echocardiography (TEE) in only $12 \%$ of patients in 1 study [10]. In another retrospective study, TR due to lead injury was detected in only $22 \%$ of patients with transthoracic echocardiography (TTE) and $45 \%$ with TEE [10]. Therefore, despite the common knowledge on the possible mechanisms of TR after cardiac device implantation, the clear evidence to explain the whole spectrum of TR could not be obtained using 2D TTE or TEE. However, with the more common usage of 3D echocardiography, insights to the definitive mechanisms of TR and relationship between TR and implanted leads can be defined more precisely [11].

The data regarding the prevalence of TR development after endocardial lead insertion is diverse [12-15]. Most of the studies were in retrospective nature and included small number of patients. Increased severity of TR has been reported to be associated with increased mortality and decreased life quality regardless of $\mathrm{LV}$ ejection fraction or PAP [13]. In a previous study, Nath et al. [16] and Höke et al. [17] reported reduced survival after PPM/ICD 
implantation in patients who had moderate or severe TR when compared to patients with no or minimal TR. Similarly, a recent retrospective study by Al-Bawardy et al. [18] reported that in addition to the trend with worsening TR, TR after cardiac device implantation was also associated with decreased survival. Our findings were also in line with the aforementioned data which clearly outline the detrimental effects of cardiac device implantation on tricuspid valve functions.

An important aspect of the change in TR and $\mathrm{RV}$ functions is the assumption of differential effects of various cardiac device types regarding to the inherent properties of those devices like thickness of coils, flexibility and number of electrodes passing through the tricuspid valve. In a previous study, involving 248 patients with ICD or PPM, Kim et al. [12] reported that ICDs cause more TR than normal PPM (32.4\% vs. $20.7 \%$, p < 0.05) and attributed this difference to less flexible and thicker nature of ICD electrodes and shocking coil causing more fibrosis at the neighboring areas of tricuspid valve. However, an important finding in our study which should be stressed is the lack of association between cardiac device types and TR and changes in RV functions, despite a statistically significant TR increase after PPM/CRT or ICD implantation. Our findings are also in line with a large scale retrospective analysis which reported a similar increase in TR with both ICD and PPM placement irrespective of the number of leads implanted or the device type used [18]. However, implantation technique, as well as the type of ICD lead might have a potential to affect those outcomes which deserves to be investigated in prospective studies with a higher number of patients included.

After PM/ICD lead implantation, tricuspid insufficiency might develop or worsen due to several proposed mechanisms including physical impingement of lead on the valve, fibrous tissue formation causing adherence and rarely perforation and entrapment of lead with valvular apparatus [19-22]. In addition, the clinical presentation of acute insult to the integrity of valvular or subvalvular apparatus might cause clinical findings in a more chronic nature depending on both baseline ventricular functions and pulmonary hypertension. An interesting finding in our study is the differential pattern of worsening in TR evaluated by color jet method or more quantitative methods like $\mathrm{VC}$ or PISA measurements. While color jet method indicated deterioration in TR in an acute pattern within the $1^{\text {st }}$ month after lead implantation, VC and PISA of the patients increased gradually throughout the follow-up period. However, the clinical significance and prognostic value of such pattern is not known.

The evaluation of the mechanisms after cardiac device implantation revealed that lead impingement, adherence, perforation and entanglement plays an important role in the formation of TR [10, 13]. In addition to those mechanisms, asynchrony due a high ratio of $\mathrm{RV}$ pacing causing deterioration in both right and right ventricular functions and tricuspid annular dilatation should not be overlooked [23]. Although some studies demonstrated beneficial effect of CRT pacing for RV functions, the number of patients with CRT is small in our study population preventing to make certain and definitive conclusions about the RV functions in those patients [24]. Despite the high pacing ratio in CRT patients, the ventricular pacing ratio is not $>10 \%$ with $\mathrm{PPM} / \mathrm{ICD}$ 's in our study population that might cause a bias effect in TR grade. Moreover, an increase in PAP can also cause TR. As demonstrated in previous studies, the increase in TR was not associated with an increase in PAP values $[12,18]$.

An important issue regarding the evaluation of TR is the quantitativity of the method we selected. Due to the retrospective nature of most of the studies evaluating this issue previously, TR jet area or ratio of regurgitation jet area to RA area based measurements were frequently used to determine the degree of TR. However, this method has the potential to underestimate TR severity especially when the RA is dilated. Moreover, in case of small difference in the systolic pressure between RA and RV, TR could be underestimated when TR jet area used. Because of those pitfalls, $\mathrm{VC}$ gained an interest in order to evaluate the severity of TR more quantitatively [23]. Therefore, different from previous studies, more quantitative methods of VC and PISA for TR was used in our study in additional to conventional TR color jet based grading which revealed an increase in PISA and VC values progressively and significantly in the follow-up period, as well as RA volumes and RV diameters. Although none of the patients in our study group additional worsening in symptomatic status or clinical right heart failure symptoms after cardiac device implantation, the follow-up period is not enough to exactly assess the change in those novel parameters for TR grading and clinical outcomes. Therefore, the association of those novel TR grading parameters should be evaluated in large scale studies which might also aid to determine the patient subgroups before clinically evident deterioration in symptomatic status. 


\section{Limitations of the study}

Our study has some limitations. First, we used 2D echocardiography for the evaluation of TR and RV functions. However, 3D echocardiography might give important clues especially regarding the exact mechanism of TR in this study population. Nevertheless, we used more quantitative parameters determined by the guidelines to evaluate TR and right heart functions optimally. Second, despite the prospective nature of our study, our study population is not large enough to determine effect of TR worsening on cardiovascular survival. Therefore, large scale prospective studies are needed in order to evaluate the clinical significance of those novel parameters on clinical outcomes. Also, our study is a single center study involving mostly the patients with decreased LV functions which is an important limitation to extrapolate those findings in a more general group of patient population.

\section{Conclusions}

In conclusion, the implantation of electrodes for PPM/CRT or ICD might cause worsening of TR and RV functions. However this effect is independent of the device implanted in our study population. Further studies are needed especially with novel imaging modalities in order to clarify the exact mechanisms of TR and RV dysfunction as well as optimal management of worsening TR after cardiac device implantation.

\section{Conflict of interest: None declared}

\section{References}

1. Mond HG, Proclemer A. The 11th world survey of cardiac pacing and implantable cardioverter-defibrillators: calendar year 2009: A World Society of Arrhythmia's project. Pacing and clinical electrophysiology. PACE, 2011; 34: 1013-1027.

2. Agarwal S, Tuzcu EM, Rodriguez ER, Tan CD, Rodriguez LL, Kapadia SR. Interventional cardiology perspective of functional tricuspid regurgitation. Circulation Cardiovascular Interventions, 2009; 2: 565-573.

3. Gibson TC, Davidson RC, DeSilvey DL. Presumptive tricuspid valve malfunction induced by a pacemaker lead: A case report and review of the literature. PACE, 1980; 3: 88-95.

4. Iskandar SB, Ann Jackson S, Fahrig S, Mechleb BK, Garcia ID. Tricuspid valve malfunction and ventricular pacemaker lead: Case report and review of the literature. Echocardiography, 2006; 23: 692-697.

5. Korkeila P, Mustonen P, Koistinen J et al. Clinical and laboratory risk factors of thrombotic complications after pacemaker implantation: A prospective study. Europace, 2010; 12: 817-824.

6. Brignole M, Auricchio A, Baron-Esquivias G et al. 2013 ESC Guidelines on cardiac pacing and cardiac resynchronization therapy: The Task Force on cardiac pacing and resynchronization therapy of the European Society of Cardiology (ESC). Developed in collaboration with the European Heart Rhythm Association (EHRA). Eur Heart J, 2013; 34: 2281-2329.

7. Quinones MA, Otto CM, Stoddard M et al. Recommendations for quantification of Doppler echocardiography: A report from the Doppler Quantification Task Force of the Nomenclature and Standards Committee of the American Society of Echocardiography. J Am Soc Echocardiogr, 2002; 15: 167-184.

8. Rudski LG, Lai WW, Afilalo J et al. Guidelines for the echocardiographic assessment of the right heart in adults: A report from the American Society of Echocardiography endorsed by the European Association of Echocardiography, a registered branch of the European Society of Cardiology, and the Canadian Society of Echocardiography. J Am Soc Echocardiogr, 2010; 23: 685-713; quiz 786-788).

9. Zoghbi WA, Enriquez-Sarano M, Foster E et al. Recommendations for evaluation of the severity of native valvular regurgitation with two-dimensional and Doppler echocardiography. J Am Soc Echocardiogr, 2003; 16: 777-802.

10. Lin G, Nishimura RA, Connolly HM, Dearani JA, Sundt TM, 3rd, Hayes DL. Severe symptomatic tricuspid valve regurgitation due to permanent pacemaker or implantable cardioverter-defibrillator leads. J Am Coll Cardiol, 2005; 45: 1672-1675.

11. de Agustin JA, Viliani D, Vieira C et al. Proximal isovelocity surface area by single-beat three-dimensional color Doppler echocardiography applied for tricuspid regurgitation quantification. J Am Soc Echocardiogr, 2013; 26: 1063-1072.

12. Kim JB, Spevack DM, Tunick PA et al. The effect of transvenous pacemaker and implantable cardioverter defibrillator lead placement on tricuspid valve function: an observational study. J Am Soc Echocardiogr, 2008; 21: 284-287.

13. Sakai M, Ohkawa S, Ueda K et al. [Tricuspid regurgitation induced by transvenous right ventricular pacing: echocardiographic and pathological observations]. J Cardiol, 1987; 17: 311-320.

14. Kucukarslan N, Kirilmaz A, Ulusoy E et al. Tricuspid insufficiency does not increase early after permanent implantation of pacemaker leads. J Cardiac Surg, 2006; 21: 391-394.

15. Paniagua D, Aldrich HR, Lieberman EH, Lamas GA, Agatston AS. Increased prevalence of significant tricuspid regurgitation in patients with transvenous pacemakers leads. Am J Cardiol, 1998; 82: 1130-1132.

16. Nath J, Foster E, Heidenreich PA. Impact of tricuspid regurgitation on long-term survival. J Am Coll Cardiol, 2004; 43: 405-409.

17. Hoke U, Auger D, Thijssen J et al. Significant lead-induced tricuspid regurgitation is associated with poor prognosis at long-term follow-up. Heart, 2014; 100: 960-968.

18. Al-Bawardy R, Krishnaswamy A, Rajeswaran J et al. Tricuspid regurgitation and implantable devices. PACE, 2015; 38: 259-266.

19. Gould L, Reddy CV, Yacob U et al. Perforation of the tricuspid valve by a transvenous pacemaker. JAMA, 1974; 230: 86-87.

20. Hill PE. Complications of permanent transvenous cardiac pacing: a 14-year review of all transvenous pacemakers inserted at one community hospitalPACE, 1987; 10: 564-570.

21. Rubio PA, al-Bassam MS. Pacemaker-lead puncture of the tricuspid valve. Successful diagnosis and treatment. Chest, 1991; 99: 1519-1520.

22. Stewart D, Leman RB, Kaiser J, Mann DL. Catheter-induced tricuspid regurgitation. Incidence and clinical significance. Chest, 1991; 99: 651-655.

23. Vaturi M, Kusniec J, Shapira Y et al. Right ventricular pacing increases tricuspid regurgitation grade regardless of the mechanical interference to the valve by the electrode. Eur J Echocardiogr, 2010; 11: 550-553.

24. Aksoy H, Okutucu S, Aytemir K et al. Improvement in right ventricular systolic function after cardiac resynchronization therapy correlates with left ventricular reverse remodeling. PACE, 2011; 34: 200-207. 